\title{
Roles of Ki67 in Breast Cancer - Important for Management?
}

\author{
CH Yip ${ }^{1 *}, \mathbf{N}$ Bhoo-Pathy ${ }^{2}, \mathrm{JM}$ Daniel $^{3}, \mathrm{YC} \mathrm{Foo}^{4}, \mathrm{AK}_{\text {Mohamed }}^{4}, \mathrm{MM} \mathrm{Abdullah}^{4}$, \\ YS Ng ${ }^{4}$, BK Yap ${ }^{4}$, R Pathmanathan ${ }^{3}$
}

\begin{abstract}
Background: The three standard biomarkers used in breast cancer are the estrogen receptor (ER), progesterone receptor (PR) and human epidermal growth factor receptor 2 (HER2). The Ki-67 index, a proliferative marker, has been shown to be associated with a poorer outcome, and despite absence of standardization of pathological assessment, is widely used for therapy decision making. We aim to study the role of the Ki-67 index in a group of Asian women with breast cancer. Materials and Methods: A total of 450 women newly diagnosed with Stage 1 to 3 invasive breast cancer in a single centre from July 2013 to Dec 2014 were included in this study. Univariable and multivariable logistic regression was used to determine the association between Ki-67 (positive defined as $14 \%$ and above) and age, ethnicity, grade, mitotic index, ER, PR, HER2, lymph node status and size. All analyses were performed using SPSS Version 22. Results: In univariable analysis, Ki -67 index was associated with younger age, higher grade, ER and PR negativity, HER2 positivity, high mitotic index and positive lymph nodes. However on multivariable analysis only tumour size, grade, PR and HER2 remained significant. Out of 102 stage 1 patients who had ER positive/PR positive/HER2 negative tumours and non-grade 3, only $5(4.9 \%)$ had a positive Ki-67 index and may have been offered chemotherapy. However, it is interesting to note that none of these patients received chemotherapy. Conclusions: Information on Ki67 would have potentially changed management in an insignificant proportion of patients with stage 1 breast cancer.
\end{abstract}

Keywords: Ki-67 index - invasive breast cancer - indications for chemotherapy

Asian Pac J Cancer Prev, 17 (3), 1077-1082

\section{Introduction}

Breast cancer is the commonest cancer in women worldwide. Currently the three standard biomarkers to determine prognosis and prediction to response to therapy is the estrogen receptor (ER), progesterone receptor (PR) and the human epidermal growth factor receptor 2 (HER2).

The use of Ki-67 index, which is a proliferative marker, as a predictive and prognostic marker in breast cancer, has been widely investigated. (Inwald et al., 2013). In general, the Ki-67 index is defined as the percentage of total number of tumour cells with nuclear staining. (Urruticoechea et al., 2005). Baseline Ki-67 index has been found to be higher in patients with triple negative breast cancer (where ER, PR and HER2 are not expressed) (Keam et al., 2011) while patients with ER positive and/or PR positive tumours had a lower Ki-67 index. (Trihia et al., 2003). Based on gene expression profiling, breast cancer can be divided into four molecular subtypes, Luminal A, Luminal B, HER2 over-expressing and Basal-like. However gene expression profiling is expensive and not practical for routine clinical practice, and surrogates for the molecular subtypes based on immunohistochemistry (IHC) evaluation of ER, PR and HER 2 have been used. While the IHC surrogates for HER 2 over-expressing subtype (ER negative, PR negative and HER 2 positive) and basal-like subtype (ER, PR and HER2 negative) are well accepted, the IHC surrogates for Luminal A and Luminal B are less well-defined with various combinations of ER, PR and HER2 suggested. The panel of experts at the St Gallen Consensus in 2011 (Goldhirsch et al., 2011) and 2013 (Goldhirsch et al., 2013) have divided the Luminal B group into a HER2 negative and a HER2 positive subtype, where the Luminal B HER2 negative subtype is a subgroup with ER positive, HER2 negative and at least one of the following: 1. Ki-67 index high ( defined as $14 \%$ or more) and PR negative or low (defined as $<20 \%$ of cells stained positive). (Table 1 )

The cut-off value of $14 \%$ was based on the work of Cheang etal who subtyped 357 patients with invasive breast cancer by gene expression profiling and together with IHC determination of hormone receptor status, HER2 status and Ki-67 index, used receiver operating

${ }^{1}$ Department of Surgery, ${ }^{3}$ Department of Pathology, ${ }^{4}$ Department of Clinical Oncology, Subang Jaya Medical Centre, Selangor, ${ }^{2}$ Department of Social and Preventive Medicine, University of Malaya, Kuala Lumpur, Malaysia *For correspondence: chenghar. yip@gmail.com 
characteristics (ROC) curves to determine that the best cut-off for K-67 index to determine between Luminal A and Luminal B was $13.25 \%$. (Cheang et al., 2009).

However the role of Ki-67 index to determine adjuvant therapy in early breast cancer is not widely practised, and $\mathrm{Ki}-67$ index is not included in most guidelines on pathology reporting of breast cancer. This is because there is currently no standardized methodology to determine the $\mathrm{Ki}-67$ index hence leading to a broad range of recommendations regarding the minimum number of cells counted to ascertain the percentage. Further variation in the method of counting ie computer aided versus human analysis leads to inter and intraoperator and laboratory variances (Dowsett et al., 2011). Because of the current interest in $\mathrm{Ki}-67$ index as a prognostic and predictive marker, we aim to study the role of Ki-67 index by investigating its association with age, ethnicity, size, grade, lymph node status, breast cancer subtype, and mitotic index in a cohort of Asian women with breast cancer. We also aim to determine the proportion of patients who potentially would have their management changed based on Ki-67 index.

\section{Materials and Methods}

\section{Patient population}

Four-hundred and fifty consecutive women newly diagnosed with Stage 1-3 breast cancer in the Subang Jaya Medical Centre (SJMC) from 1st July 2013 to 31st December 2014 were included. The SJMC is a private hospital in an urban setting in Klang Valley in Malaysia. We excluded women with Stage 4 breast cancer because the numbers were low. The study is approved by the institutional ethics committee.

\section{Immunohistochemistry}

ER, PR and HER2 were determined by immunohistochemistry (IHC) staining of the tumour. ER and PR were considered negative if staining of the tumour cell nuclei was $1 \%$ or less, regardless of staining intensity. HER2 was assessed through IHC and based on the staining intensity and percentage, which was classified as 0 (negative) $1+, 2+$ and $3+$ according to the criteria set by DAKO. Only a report of negative or 1+ was taken as HER2 negative. Silver in situ hybridization was carried out when there was a report of $2+$, and a ratio of HER2 gene to chromosome 17 of $>2.2$ confirmed that HER2 was positive.

Ki-67 index was recorded as the percentage of positively staining malignant cells using the anti-human Ki-67 monoclonal MIB1 which is one of the most commonly used antibodies and considered as the gold standard. The Ki-67 percentage score or Ki-67 index is defined as the percentage of positively stained tumour cells among the total number of malignant cells assessed, and routinely 100 malignant cells in a representative section of the tumour were counted.

All the four biomarkers were evaluated by two pathologists in a single pathology laboratory, hence reducing any inter-observer or inter-laboratory variation

\section{Statistical analysis}

Univariable and multivariable logistic regression analysis was used to determine the association between $\mathrm{Ki}$ 67 index (positive was taken as $14 \%$ and above) and each of the following variables - age (continuous), ethnicity (Chinese, Malays, Indians and Others), grade (1 to 3), mitotic index (0-7, 8-14, 15 and above), ER (positive or negative), PR (positive or negative) HER2 (positive or negative), lymph node status ( $\mathrm{N} 0=$ no nodes involved, N1=1-3 lymph nodes involved, N2=4-9 lymph nodes involved, N3=10 or more lymph nodes involved), Stage $(1,2$ or 3$)$, and size $(2 \mathrm{~cm}$ or less, $>2 \mathrm{~cm})$. All analysis was done using SPSS Version 22.

\section{Results}

The median age at diagnosis was 51 years. The median tumour size was $20 \mathrm{~mm}$. The majority of the patients were Chinese $(89.5 \%)$ and most patients presented with Stage 1 and 2 disease (40.2\% and $38.7 \%$ respectively). Most patients presented with Grade $2(48.4 \%)$, ER positive (70.7\%), PR positive $(63.8 \%)$, and HER2 negative (75.1\%) tumors. Using the definition of molecular subtypes as defined by the St Gallen's Consensus (Table 2), the majority of patients (37.2\%) had Luminal A breast cancer while $16.3 \%$ had triple negative breast cancer.

Ki-67 index absolute values ranged from 1 to 80, with a mean of 16 and a median of 10 . The median values were significantly higher in women less than 40 years old, higher grade, higher mitotic index, ER negative, PR negative and HER2 positive. There did not seem to be a significant association with size or number of involved axillary lymph nodes. (Table 3) There was a trend for median values to be higher in Indians compared to Malays and Chinese although this was not significant.

Using $14 \%$ as the cut-off value for Ki-67 index, $43.4 \%$ of patients were $\mathrm{Ki}-67$ index positive. There was no association between ethnicity and Ki-67 index although Indian patients were more likely to have a positive Ki-67 index. In univariable analysis, Ki-67 index positivity was significantly associated with a younger age, higher grade, ER and PR negativity, HER2 positivity, larger tumour size, a high mitotic index and positive lymph nodes. However in multivariable analysis, only tumour size, grade, PR and HER2 remained significant. Mitotic index was not found to be significant in the multivariable analysis, and a step-wise adjustment indicated that the initial association between mitotic index and Ki-67 index was completely explained by the underlying differences in tumour grade. (Table 4)

In the latest Malaysian Clinical Practice Guidelines for Management of Breast Cancer, adjuvant chemotherapy is indicated in women with one or more positive lymph nodes, ER and PR negative tumours, HER2 positivity, tumour larger than $2 \mathrm{~cm}$ or in grade 3 tumours. Ki-67 positivity is not considered a criteria in decision-making for chemotherapy. Using this criteria, of the 450 patients, $77.6 \%$ would have required chemotherapy. Notably, out of 102 Stage 1 patients who have ER positive PR positive HER2 negative tumours and non-Grade 3, only 5 (4.9\%) had a Ki-67 index of more than $14 \%$. It is interesting to 
Table 1. Breast Cancer Subtypes (St Gallen Consensus)

\begin{tabular}{ll}
\hline \multicolumn{1}{c}{ Breast Cancer Subtype } & \multicolumn{1}{c}{ Definition } \\
\hline \multirow{2}{*}{ Luminal A } & ER positive, PR positive, HER2 negative \\
& Ki-67 index low (defined as <14\%) \\
& Luminal B (HER2 negative) \\
& ER positive, HER2 negative and at one of: \\
& Ki-67 index high (defined as 14\% and above) \\
Luminal B & PR negative or low (defined as <20\%) \\
& Luminal B (HER2 positive) \\
& ER positive, HER2 over-expressed or amplified, any Ki-67 index, Any PR \\
HER2 over-expressing & HER2 over-expressed or amplified, ER and PR negative \\
Triple negative & ER and PR negative, HER2 negative \\
\hline
\end{tabular}

*Reference: Goldhirsch etal 2013 (Reference 16)

Table 2. Characteristics of 450 Patients with Stage 1-3 Breast Cancer

\begin{tabular}{|c|c|c|}
\hline & No & $\%$ \\
\hline \multicolumn{3}{|l|}{ Ethnicity } \\
\hline Chinese & 403 & 89.6 \\
\hline Malay & 23 & 5.1 \\
\hline Indian & 16 & 3.6 \\
\hline Others & 8 & 1.8 \\
\hline \multicolumn{3}{|l|}{ Stage } \\
\hline 1 & 192 & 42.7 \\
\hline 2 & 182 & 40.4 \\
\hline 3 & 76 & 16.9 \\
\hline \multicolumn{3}{|l|}{ Grade } \\
\hline 1 & 47 & 10.9 \\
\hline 2 & 211 & 48.7 \\
\hline 3 & 175 & 40.4 \\
\hline \multicolumn{3}{|l|}{ Size } \\
\hline $2 \mathrm{~cm}$ or less & 257 & 57.1 \\
\hline$>2 \mathrm{~cm}$ & 193 & 42.9 \\
\hline \multicolumn{3}{|l|}{ Mitotic index } \\
\hline 1 to 7 & 252 & 59.4 \\
\hline 8 to 14 & 109 & 25.7 \\
\hline 15 and above & 63 & 14.9 \\
\hline \multicolumn{3}{|l|}{ ER } \\
\hline Negative & 133 & 22.6 \\
\hline Positive & 317 & 70.4 \\
\hline \multicolumn{3}{|l|}{$\mathrm{PR}$} \\
\hline Negative & 161 & 35.8 \\
\hline Positive & 289 & 64.2 \\
\hline \multicolumn{3}{|l|}{ HER2 } \\
\hline Negative & 341 & 75.8 \\
\hline Positive & 109 & 24.2 \\
\hline \multicolumn{3}{|l|}{ Lymph nodes } \\
\hline 0 & 274 & 61.9 \\
\hline 1 to 3 & 96 & 1.7 \\
\hline 4 to 9 & 39 & 8.8 \\
\hline 10 and more & 34 & 7.7 \\
\hline \multicolumn{3}{|l|}{ Stage } \\
\hline 1 & 192 & 42.7 \\
\hline 2 & 182 & 40.4 \\
\hline 3 & 76 & 16.9 \\
\hline \multicolumn{3}{|l|}{ Molecular subtype } \\
\hline Luminal A & 174 & 38.7 \\
\hline Luminal B HER2 negative & 92 & 20.4 \\
\hline Luminal B HER2 positive & 59 & 13.1 \\
\hline HER 2 over-expressing & 56 & 11.1 \\
\hline Triple negative breast cancer & 75 & 16.7 \\
\hline
\end{tabular}

Table 3. Differentiation of Ki-67 index by Analysis of Yariance (ANOVA) in 450 Patients

\begin{tabular}{|c|c|c|c|}
\hline \multicolumn{2}{|c|}{ Absolute median Ki-67 index } & SD & P Value \\
\hline \multicolumn{4}{|l|}{ Ethnicity } \\
\hline Chinese & 10 & 15.2 & 0.59 \\
\hline Malays & 10 & 15.3 & \\
\hline Indians & 15 & 15 & \\
\hline Others & 12.5 & 12.5 & \\
\hline \multicolumn{4}{|l|}{ Age } \\
\hline$<40$ & 19 & 19.3 & $0.00^{*}$ \\
\hline 40 and above & 10 & 14.9 & \\
\hline \multicolumn{4}{|l|}{ Size } \\
\hline $2 \mathrm{~cm}$ and below & 10 & 14.5 & 0.07 \\
\hline$>2 \mathrm{~cm}$ & 15 & 16.1 & \\
\hline \multicolumn{4}{|c|}{ Axillary lymph nodes involved } \\
\hline NO & 10 & 15.1 & 0.11 \\
\hline N1 & 11.5 & 15.2 & \\
\hline N2 & 12.5 & 17.5 & \\
\hline N3 & 18 & 15.9 & \\
\hline \multicolumn{4}{|l|}{ Grade } \\
\hline 1 & 5 & 3.4 & $0.00^{*}$ \\
\hline 2 & 8 & 12.7 & \\
\hline 3 & 20 & 17.3 & \\
\hline \multicolumn{4}{|l|}{ ER } \\
\hline Negative & 20 & 19.8 & $0.00^{*}$ \\
\hline Positive & 9.5 & 11.1 & \\
\hline \multicolumn{4}{|l|}{ PR } \\
\hline Negative & 18 & 18.8 & $0.00^{*}$ \\
\hline Positive & 8 & 11 & \\
\hline \multicolumn{4}{|l|}{ HER2 } \\
\hline Negative & 10 & 15.4 & $0.00^{*}$ \\
\hline Positive & 17 & 14.5 & \\
\hline \multicolumn{4}{|l|}{ Mitotic index } \\
\hline 0.7 & 8 & 12.9 & $0.00 *$ \\
\hline $8-14$ & 15 & 13.9 & \\
\hline$\geq 15$ & 21 & 19.2 & \\
\hline
\end{tabular}

* Statistically Significant

note that none of these patients received chemotherapy.

\section{Discussion}

The ASCO-CAP (American Society of Clinical Oncologists - College of American Pathologists) guidelines clearly lay out what is considered to be ER, PR and HER2 positive and that quality assurance is of the utmost importance. (Hammond et al., 2010; Wolff et al., 
Table 4. Association Between Demographic / Tumor Characteristics with Ki67 Status in 450 Patients with Stage I - stage III Breast Cancer

\begin{tabular}{|c|c|c|c|c|}
\hline & Ki-67 negative & Ki-67 positive & $\begin{array}{l}\text { Univariable odds ratio for } \\
\text { positive Ki-67 (95\% CI) }\end{array}$ & $\begin{array}{l}\text { Multivariable odds ratio for } \\
\text { positive Ki-67 (95\% CI) }\end{array}$ \\
\hline Median age (years) & 51 & 51 & $0.98(0.87-1.00)^{*}$ & $0.98(0.96-1.00)$ \\
\hline \multicolumn{5}{|l|}{ Ethnicity } \\
\hline Chinese & 236 & 167 & 1.00 & 1.00 \\
\hline Malays & 12 & 11 & $1.29(0.57-3.01)$ & $0.90(0.34-2.40)$ \\
\hline Indians & 8 & 8 & $1.41(0.52-3.84)$ & $1.16(0.33-4.06)$ \\
\hline Others & 4 & 4 & $1.410 .35-5.73)$ & $1.20(0.26-5.53)$ \\
\hline Median tumour size $(\mathrm{cm})$ & 2.0 & 2.25 & $1.29(1.11-1.49)^{*}$ & $1.23(1.03-1.47)^{*}$ \\
\hline \multicolumn{5}{|c|}{ Axillary lymph nodes involved } \\
\hline No & 173 & 101 & 1.00 & 1.00 \\
\hline N1 & 48 & 48 & $1.71(1.7-2.74)^{*}$ & $1.55(0.88-2.73)$ \\
\hline $\mathrm{N} 2$ & 19 & 20 & $1.80(0.92-3.54)$ & $1.37(0.62-3.05)$ \\
\hline $\mathrm{N} 3$ & 14 & 20 & $2.45(1.18-5.06)^{*}$ & $1.29(0.53-3.14)$ \\
\hline \multicolumn{5}{|l|}{ Grade } \\
\hline 1 & 45 & 2 & $0.02(0.05-0.09)^{*}$ & $0.05(0.01-0.23) *$ \\
\hline 2 & 150 & 61 & $0.20(0.13-0.31)^{*}$ & $0.25(0.12-0.49)^{*}$ \\
\hline 3 & 58 & 117 & 1.00 & 1.00 \\
\hline \multicolumn{5}{|l|}{ ER } \\
\hline Negative & 42 & 91 & 1.00 & 1.00 \\
\hline Positive & 218 & 99 & $0.21(0.14-0.32)^{*}$ & $0.85(0.40-1.81)$ \\
\hline \multicolumn{5}{|l|}{ PR } \\
\hline Negative & 54 & 107 & 1.00 & 1.00 \\
\hline Positive & 206 & 83 & $0.20(0.13-0.31)^{*}$ & $0.42(0.21-0.86)^{*}$ \\
\hline \multicolumn{5}{|l|}{ HER2 } \\
\hline Negative & 226 & 115 & 1.00 & 1.00 \\
\hline Positive & 34 & 75 & $4.33(2.73-6.89)^{*}$ & $3.01(1.74-5.20)^{*}$ \\
\hline \multicolumn{5}{|l|}{ Mitotic index } \\
\hline 0.7 & 181 & 71 & 1.00 & 1.00 \\
\hline $8-14$ & 47 & 62 & $3.36(2.11-5.37)^{*}$ & $0.58(0.28-1.20)$ \\
\hline$\geq 15$ & 19 & 44 & $5.90(3.23-10.80)^{*}$ & $0.35-2.01)$ \\
\hline
\end{tabular}

* Statistically significant

2014) The role of Ki-67 index as a prognostic marker is well-studied though significant issues remain regarding its predictive utility and validity, and these issues have to be addressed before it can be used routinely in clinical practice.

In spite of it not being a recommended routine marker in breast pathology, it continues to be reported in several centres, and is used as a marker for decision making in guidelines such as the St Gallens consensus. (Goldhirsch et al., 2013) The Ki-67 index is also one of the variables included in the web-based online prognostic model, PREDICT. PREDICT was developed in the United Kingdom, and had been recommended to aid prognostication and selection of patients with early breast cancer for adjuvant chemotherapy. (Wishart et al., 2010) However, the added prognostic value of $\mathrm{Ki}-67$ index in the PREDICT program is yet to be externally validated due to a lack of information on $\mathrm{Ki}-67$ index in the appropriate population. A recent publication cautions oncologists not to rely on the use of Ki67 for patient with early breast cancer and only 1-3 positive lymph nodes (Andre et al., 2015).

The histopathological factors associated with Ki-67 index positivity and higher Ki-67 absolute value, such as a higher grade, ER and PR negativity, HER 2 positivity and high mitotic index seen in this study is also comparable to other studies. (Trihia et al., 2003; Wiesner et al., 2009; Inwald et al., 2013). A larger tumour and lymph node positivity, which are time-dependent factors, are also associated with a positive Ki-67 index and higher absolute Ki-67 index values. In multivariable analysis, lymph node status, ER status and mitotic index were no longer significantly associated with Ki-67 index.

A meta-analysis of 46 studies including 12155 patients evaluated the impact of Ki-67 index on disease-free and overall survival, and found that Ki-67 index positivity (according to the cut-off points as defined by each study) was associated with a higher probability of relapse in all patients. What is interesting is the wide variation in the cut-off value in the studies, which ranged from $3.5 \%$ to $32 \%$, with the majority of studies using $10 \%$ as an arbitrary cut-off value, because that was the median value of Ki-67 index. (de Azambuja et al., 2007). Spyratos etal compared five different cut-offs $(10 \%, 15 \%, 17 \%$, $20 \%$ and $25 \%$ ) with other proliferative markers such as mitotic index and grade, and decided that a $\mathrm{Ki}-67$ index 
cut-off of $25 \%$ was optimal to identify patients sensitive to chemotherapy protocols, and with a cut-off of $10 \%$, few tumours with low proliferation would be misclassified. (Spyratos et al., 2002). The PREDICT model defined a positive Ki-67 index as being more than $10 \%$. (Wishart et al., 2010). However the best evidence for the cut-off value is from a study by Cheang etal, who used gene expression profiling to determine that the best $\mathrm{Ki}-67$ index cut-off to differentiate between Luminal A and Luminal B HER2 negative was $13.25 \%$. (Cheang et al., 2009). Notably, the median value of Ki-67 index in this study was 15 , and hence the cut-off value of $14 \%$ as advised by the $\mathrm{St}$ Gallens Consensus is highly likely to identify the more proliferative tumours (Goldhirsch et al., 2013).

A recent study had demonstrated that the IHC4 (based on ER, PR, HER2 and Ki-67 index) is equivalent to genomic profiling (ie Oncotype DX and Mammoprint) in predicting outcome in estrogen-receptor positive breast cancers (Cuzick et al., 2011). However calculation of the IHC4 required quantitative estimates of the ER, PR and Ki-67 index in percentages. The IHC4 score classifies tumours into low, intermediate and high-risk. It is important to note that these recurrence scores were designed for ER positive, HER2 negative and lymph node negative tumours, i.e. the group where the need for chemotherapy is equivocal. Compared with the cost of genomic profiling, it has been suggested that testing of Ki-67 index would be resource-saving and economical especially if done concurrently with other biomarkers which are already in standard clinical practice.

Besides being a prognostic marker, Ki-67 index has been shown to be predictive of the response to neoadjuvant chemotherapy, whereby a higher Ki-67 index is associated with higher pathologic complete response rate. It has been suggested that tumours with a Ki-67 index above $25 \%$ may be better candidates for neoadjuvant chemotherapy. (Nishimura et al., 2010). Measurement of Ki-67 index before and after a 2-week course of pre-surgical hormone therapy found that higher levels of Ki-67 index after the course of hormone therapy was statistically significantly associated with a lower recurrence-free survival (Dowsett et al., 2007).

Decision-making on adjuvant therapy depends on institutional guidelines, as well as international guidelines. Women decide on adjuvant therapy based on their perception of risks and benefits of adjuvant therapy with guidance from their treating physician. It has been suggested that women with a positive K-67 index should be offered chemotherapy because of poorer outcomes. However it is noted that if Ki-67 positivity is used for decision-making, only $4.9 \%$ of the "good" prognosis patients, i.e. node negative, $2 \mathrm{~cm}$ or smaller, ER and/or PR positive, HER 2 negative, and non-Grade 3, had Ki-67 index levels $14 \%$ and above, and potentially would have been advised for adjuvant chemotherapy. However, none of these patients in the current study received adjuvant chemotherapy. This indicates that the oncologists do not regard Ki-67 index positivity as a sole criteria for adjuvant chemotherapy administration.

In conclusion, The absence of standardization of Ki-67 index requires further research before it can be accepted
Roles of Ki67 in Breast Cancer - Important for Management?

as a routine prognostic or predictive marker. (Jonat and Arnold, 2011) Further analysis using validated methods is necessary before its widespread adoption and only a complete standardization of tissue handling and processing will improve the value of $\mathrm{Ki}-67$ index as a clinically useful marker (Dowsett et al., 2007).

Despite all these reservations, the role of Ki-67 index in decision making seems to be established in several treatment guidelines and prediction models. Based on the current study, it appear that in the absence of other pathologically adverse features, information on Ki-67 index would have little impact on chemotherapy decisionmaking.

\section{References}

Malaysian Clinical Practice Guidelines for the Management of Breast Cancer Nov 2010 http://www.moh.gov.my.

Andre F, Arnedos M, Goubar A, et al (2015). Ki67-no evidence for its use in node-positive breast cancer. Nat Rev Clin Oncol.

Cheang MC, Chia SK, Voduc D, et al (2009). Ki67 index, HER2 status, and prognosis of patients with luminal B breast cancer. J Natl Cancer Inst, 101, 736-50.

Cuzick J, Dowsett M, Pineda S, et al (2011). Prognostic value of a combined estrogen receptor, progesterone receptor, $\mathrm{Ki}-67$, and human epidermal growth factor receptor 2 immunohistochemical score and comparison with the Genomic Health recurrence score in early breast cancer. $J$ Clin Oncol, 29, 4273-8.

de Azambuja E, Cardoso F, de Castro G, Jr., et al (2007). Ki-67 as prognostic marker in early breast cancer: a meta-analysis of published studies involving 12,155 patients. Br J Cancer, 96, 1504-13.

Dowsett M, Nielsen TO, A'Hern R, et al (2011). Assessment of Ki67 in breast cancer: recommendations from the International Ki67 in Breast Cancer working group. J Natl Cancer Inst, 103, 1656-64.

Dowsett M, Smith IE, Ebbs SR, et al (2007). Prognostic value of Ki67 expression after short-term presurgical endocrine therapy for primary breast cancer. J Natl Cancer Inst, 99 , 167-70.

Goldhirsch A, Winer EP, Coates AS, et al (2013). Personalizing the treatment of women with early breast cancer: highlights of the St Gallen International Expert Consensus on the Primary Therapy of Early Breast Cancer 2013. Ann Oncol, 24, 2206-23.

Goldhirsch A, Wood WC, Coates AS, et al (2011). Strategies for subtypes--dealing with the diversity of breast cancer: highlights of the st. gallen international expert consensus on the primary therapy of early breast cancer 2011. Ann Oncol, 22, 1736-47.

Hammond ME, Hayes DF, Dowsett M, et al (2010). American Society of Clinical Oncology/College Of American Pathologists guideline recommendations for immunohistochemical testing of estrogen and progesterone receptors in breast cancer. J Clin Oncol, 28, 2784-95.

Inwald EC, Klinkhammer-Schalke M, Hofstadter F, et al (2013). $\mathrm{Ki}-67$ is a prognostic parameter in breast cancer patients: results of a large population-based cohort of a cancer registry. Breast Cancer Res Treat, 139, 539-52.

Jonat W, Arnold N (2011). Is the Ki-67 labelling index ready for clinical use? Ann Oncol, 22, 500-2.

Keam B, Im SA, Lee KH, et al (2011). Ki-67 can be used for further classification of triple negative breast cancer into two subtypes with different response and prognosis. Breast Cancer Res, 13, 22. 
Cheng-Har Yip et al

Nishimura R, Osako T, Okumura Y, et al (2010). Clinical significance of Ki-67 in neoadjuvant chemotherapy for primary breast cancer as a predictor for chemosensitivity and for prognosis. Breast Cancer, 17, 269-75.

Spyratos F, Ferrero-Pous M, Trassard M, et al (2002). Correlation between MIB-1 and other proliferation markers: clinical implications of the MIB-1 cutoff value. Cancer, 94, 2151-9.

Trihia H, Murray S, Price K, et al (2003). Ki-67 expression in breast carcinoma: its association with grading systems, clinical parameters, and other prognostic factors--a surrogate marker? Cancer, 97, 1321-31.

Urruticoechea A, Smith IE, Dowsett M (2005). Proliferation marker Ki-67 in early breast cancer. J Clin Oncol, 23, 7212-20.

Wiesner FG, Magener A, Fasching PA, et al (2009). Ki-67 as a prognostic molecular marker in routine clinical use in breast cancer patients. Breast, 18, 135-41.

Wishart GC, Azzato EM, Greenberg DC, et al (2010). PREDICT: a new UK prognostic model that predicts survival following surgery for invasive breast cancer. Breast Cancer Res, 12, R1.

Wolff AC, Hammond ME, Hicks DG, et al (2014). Recommendations for human epidermal growth factor receptor 2 testing in breast cancer: American society of clinical oncology/college of American pathologists clinical practice guideline update. Arch Pathol Lab Med, 138, 241-56. 